\title{
Artículo
}

\section{Adquisición de competencias en el Grado de Turismo mediante el aprendizaje basado en estudios de caso}

\author{
Pablo Juan Cárdenas-García*, Juan Ignacio Pulido-Fernández e Isabel Carrillo-Hidalgo \\ Departamento de Economía, Universidad de Jaén, Jaén, España
}

\section{INFORMACIÓN DEL ARTÍCULO}

\section{Historia del artículo:}

Recibido el 7 de enero de 2015

Aceptado el 9 de mayo de 2015

On-line el 12 de agosto de 2015

\section{Palabras clave:}

Adquisición de competencias

Estudio de caso

Universidad

Grado de Turismo

\begin{abstract}
R E S U M E N
El mercado laboral demanda profesionales que posean habilidades más allá de los contenidos de los propios planes de estudios: las denominadas competencias clave o transversales, lo que implica que los agentes que intervienen en el proceso de enseñanza-aprendizaje del alumnado tienen que desarrollar metodologías docentes que permitan una adecuada adquisición de dichas competencias. Este artículo tiene como objetivo analizar si el estudio de caso favorece más el desarrollo de competencias transversales que otras metodologías tradicionales. Esta metodología se ha aplicado en las asignaturas de Economía Aplicada impartidas en el Grado de Turismo de la Universidad de Jaén. Así, mediante el uso de técnicas estadísticas inferenciales aplicadas a una muestra de alumnos dividida en 2 grupos (grupo de control: aquellos que han seguido métodos de docencia tradicionales; grupo experimental: aquellos que han utilizado el estudio de caso como metodología docente), los resultados obtenidos apoyan que existen diferencias significativas, en cuanto a la adquisición de las competencias claves o transversales, dependiendo de la metodología docente utilizada. Por tanto, la experiencia descrita aporta evidencias de que la metodología del estudio de caso podría mejorar la adquisición de competencias transversales del alumnado que cursa el Grado de Turismo en la Universidad de Jaén.
\end{abstract}

(C) 2015 Instituto de Ciencias de la Educación de la Universidad de Oviedo. Publicado por Elsevier España, S.L.U. Este es un artículo Open Access bajo la licencia CC BY-NC-ND (http://creativecommons.org/licenses/by-nc-nd/4.0/).

\section{Skills acquisition in Tourism Degree through case-based learning}

\section{A B S T R A C T}

The labor market requires professionals with skills that go beyond the contents of the curriculum itself, the so-called key or cross curricular skills. This implies that those involved in the teaching-learning process of students must develop teaching methodologies that enable the appropriate acquisition of those skills. This paper aims to analyze whether the case study favors the development of generic skills more than traditional methodologies. This methodology has been applied to courses of Applied Economics taught in the Tourism Degree at the University of Jaen. Thus, by using inferential statistics applied to a sample of students divided into two groups (control group: those who were taught using traditional teaching methods; experimental group: those who have used case studies as their teaching methodology). The results obtained support that there are significant differences regarding the acquisition of key or cross curricular skills depending on the teaching methodology used. Therefore, the experience described here provides evidence that case study methodology could improve the acquisition of cross curricular skills by students taking the Tourism Degree at the University of Jaen.

(c) 2015 Instituto de Ciencias de la Educación de la Universidad de Oviedo. Published by Elsevier España, S.L.U. This is an open access article under the CC BY-NC-ND license (http://creativecommons.org/licenses/by-nc-nd/4.0/).

\section{Introducción}

* Autor para correspondencia. Departamento de Economía, Universidad de Jaén Campus de Las Lagunillas, s/n, Edificio D3 Despacho 231 C.P. 23071, Jaén (España). Correo electrónico: pcgarcia@ujaen.es (P.J. Cárdenas-García).
Existe cierta unanimidad acerca de las competencias que, actualmente, se les exigen a los titulados universitarios, en la medida en que, independientemente de la titulación cursada, las empresas 
demandan que los futuros profesionales posean nuevas habilidades, tan importantes como los contenidos de los propios planes de estudios, entre las que destacan el trabajo en equipo, la identificación de problemas, la creatividad o la capacidad de liderazgo.

En esta línea, el Grado de Turismo, bajo el nuevo escenario que ha establecido el Espacio Europeo de Educación Superior (EEES), ha formulado los objetivos educativos en términos de las denominadas "competencias». El concepto "competencia» engloba no solo las capacidades requeridas para el ejercicio de una actividad profesional, sino también un conjunto de comportamientos considerados necesarios para el pleno desempeño de la ocupación (ANECA, 2004).

Además, de forma concreta en el sector turístico, de cara al horizonte temporal 2015-2020, y como consecuencia de las nuevas tendencias que ganarán fuerza en dicho sector, surgen una serie de perfiles profesionales emergentes, así como necesidades de cambios en perfiles tradicionales, que necesitan un importante proceso de adaptación en las competencias de los profesionales del sector (SEPE, 2011).

La metodología docente tiene que tener en cuenta estos nuevos retos a los que se enfrenta el sector turístico. Sin embargo, los métodos tradicionales de docencia universitaria, en los que el profesor decide la información que el alumnado recibe, convierten a este en un mero recipiente de información (García, 2010), lo que ha demostrado que estos sistemas de enseñanza preparan para superar un examen, pero no capacitan para enfrentarse a una situación real. En consecuencia, se identifican dificultades en el proceso de aprendizaje (Oliveira, Mazera, Guillaumon y Fernández, 2014), dado que aún no se han desarrollado competencias relacionadas con la innovación, la comunicación oral, el trabajo en equipo o la capacidad de análisis -denominadas competencias clave o transversales-, que son demandadas a los futuros profesionales, cada vez en mayor medida, por empresas y organizaciones del sector turístico.

Por tanto, en este nuevo contexto -caracterizado por la necesidad de que el alumnado adquiera una serie de competencias transversales y nuevas habilidades para adaptarse a los nuevos perfiles del sector turístico-, surge la necesidad de desarrollar modelos innovadores de docencia que permitan mejorar el proceso de aprendizaje de cara a mejorar la inserción en el mercado laboral.

Sin embargo, la impartición de los contenidos de las asignaturas en el Grado de Turismo de la Universidad de Jaén se ha venido desarrollando, habitualmente, con métodos tradicionales de docencia universitaria. Esto puede traducirse, como ya se ha apuntado, en que no se adquieran, con total garantía, habilidades tan importantes como el trabajo en equipo, la creatividad o la capacidad de liderazgo.

Por tanto, los docentes responsables de impartir las asignaturas de Economía Aplicada en el Grado de Turismo de la Universidad de Jaén, conscientes de la necesidad de que el alumnado se adapte a los nuevos perfiles profesionales que demanda el sector turístico, se propusieron desarrollar formas alternativas de docencia, con la intención de que se adquiriera, en mayor medida, una serie de competencias clave en determinadas áreas de la actividad turística.

Para cumplir con este objetivo, en primer lugar, era necesario elegir una metodología de docencia innovadora, que permitiera cumplir con la misión planteada - mejora de la adquisición de competencias y nuevas habilidades por parte del alumno-.

En este sentido, la representación de una situación de la realidad como base para la reflexión y el aprendizaje se ha convertido en una herramienta alternativa para la docencia universitaria, de cara a la consecución de estas competencias. En efecto, el planteamiento de un estudio de caso es siempre una oportunidad de aprendizaje significativo y trascendente, en la medida en que quienes participan en su análisis logran involucrarse y comprometerse, tanto en la discusión del caso como en el proceso grupal para su reflexión (Martínez, 2006; Moen, 2006).
Además, diversos informes (CGC, 1998; FCD, 2004; FUS, 2004; Universia, 2007) han señalado la necesidad de introducir, de forma paulatina, el método del estudio de caso como metodología docente, debido a que permite al alumnado adquirir las competencias anteriormente descritas.

Por tanto, el objetivo del presente artículo es analizar si la puesta en marcha de una metodología de aula basada en el estudio de caso favorece la adquisición de las competencias transversales de la titulación en mayor medida que otras metodologías, en una muestra de alumnos del Grado de Turismo.

Así, se establece como hipótesis de esta investigación que la metodología docente basada en el estudio de caso, aplicada a las asignaturas de Economía Aplicada del Grado de Turismo, mejora el rendimiento del alumnado con respecto a las promociones predecesoras, con relación a la adquisición de las competencias clave o transversales, siguiendo la misma línea que los estudios previos, expuestos con anterioridad, que defienden esta postura.

\section{Método}

\section{Diseño}

Para comprobar si el estudio de caso mejora la adquisición de competencias transversales por parte del alumno, se ha utilizado un diseño de cohortes en el que los alumnos del Grado de Turismo han sido clasificados en 2 grupos, según se haya utilizado con ellos, o no, dicha metodología docente.

Se ha utilizado el estudio de caso, como prueba piloto, en una promoción académica completa -alumnado que inició sus estudios en el curso académico 2010-2011 y lo finalizó en el curso académico 2013-2014-, en las 3 asignaturas de Economía Aplicada impartidas en el Grado de Turismo de la Universidad de Jaén.

Por tanto, comparando los resultados obtenidos por esta promoción académica con los obtenidos por la promoción anterior, que utilizaron sistemas tradicionales de docencia -en los que el profesor decide la información que se recibe, facilitando un manual de referencia, y el alumnado demuestra los conocimientos adquiridos mediante un examen, para ser recompensando con un nota a las respuestas aportadas (García, 2010)-, se podría obtener evidencia acerca de si esta metodología docente innovadora ha podido mejorar el proceso de aprendizaje con relación a la adquisición de las denominadas competencias clave o transversales y, por consiguiente, si ello mejora notablemente la inserción en el mercado laboral de los alumnos.

\section{Participantes}

Para la presente investigación se ha podido disponer de información de un total de 56 estudiantes en una muestra formada por 2 grupos (grupo de control: alumnado que ha seguido métodos de docencia tradicionales y grupo experimental: alumnado que ha utilizado el estudio de caso como metodología docente) cuyas características básicas se detallan en la tabla 1. El grupo de control está por formado por 32 integrantes, que han seguido métodos de docencia tradicionales (durante la promoción académica iniciada en el curso 2009-2010 y finalizada en el curso 2012-2013). El grupo experimental está formado por 24 integrantes, que han seguido como método de docencia el estudio de caso en las asignaturas de Economía Aplicada (durante la promoción académica iniciada en el curso 2010-2011 y finalizada en el curso 2013-2014). La muestra incluye a todo el alumnado que cursó durante dicho período las 3 asignaturas implicadas en la presente investigación y realizó las prácticas curriculares de la titulación.

Además, para la evaluación del alumnado, ha sido necesaria la participación tanto de los profesores encargados de impartir las 3 
Tabla 1

Características de la muestra

\begin{tabular}{|c|c|c|c|c|c|}
\hline \multirow[t]{2}{*}{ Variables } & & \multicolumn{2}{|c|}{ Grupo control } & \multicolumn{2}{|c|}{ Grupo experimental } \\
\hline & & $\mathrm{N}$ & $\%$ & $\mathrm{~N}$ & $\%$ \\
\hline \multirow[t]{2}{*}{ Sexo } & Hombre & 11 & 34,38 & 10 & 41,67 \\
\hline & Mujer & 21 & 65,63 & 14 & 58,33 \\
\hline \multirow[t]{2}{*}{ Nacionalidad } & Español & 30 & 93,75 & 23 & 95,83 \\
\hline & Extranjero & 2 & 6,25 & 1 & 4,17 \\
\hline Edad de finalización de los & $18-21$ & 19 & 59,38 & 17 & 70,83 \\
\hline \multirow[t]{3}{*}{ estudios } & $22-25$ & 7 & 21,88 & 4 & 16,67 \\
\hline & $26-29$ & 5 & 15,63 & 1 & 4,17 \\
\hline & $27-50$ & 1 & 3,13 & 2 & 8,33 \\
\hline Número medio de veces & 1 & 25 & 78,13 & 20 & 83,33 \\
\hline \multirow[t]{2}{*}{ matriculado en las asignaturas } & 2 & 5 & 15,63 & 3 & 12,50 \\
\hline & $>3$ & 2 & 6,25 & 1 & 4,17 \\
\hline Total & & 32 & 100,0 & 24 & 100,0 \\
\hline
\end{tabular}

asignaturas implicadas -3 docentes-, como de los tutores externos de las empresas o instituciones en las que estos han realizado las prácticas curriculares del plan de estudios -17 tutores externos-. En este sentido, ha sido total el nivel de participación de los responsables implicados en la evaluación, tanto de docentes como de tutores externos. Además, ambos grupos han sido evaluados por las mismas personas, dado que los docentes encargados de impartir las asignaturas han sido los mismos en ambas promociones y los tutores externos tampoco han cambiado al haber realizado las prácticas externas, ambas promociones, en las mismas instituciones.

\section{Programa de intervención}

Una vez diseñados los diferentes estudios de caso por parte de los docentes, se procedió a explicar al alumnado la metodología docente que se iba a seguir en la correspondiente asignatura, exponiendo los criterios de evaluación, así como la estructura que seguirían los estudios de caso (se incluye un ejemplo de una de estas actividades en el Apéndice A).

En primer lugar, se incluye una pregunta motriz que permite describir de forma rápida la situación que se desea analizar. En segundo lugar, se presenta el escenario que detalla la situación que hay que analizar, indicando el contexto que se pretende que forme parte activa del desarrollo del caso, así como fuentes de información, variables y bases de datos adicionales que permitan obtener evidencia sobre el caso objeto de estudio.

En tercer lugar, se detallan las actividades periódicas, las cuales consisten en 2 entregables, relacionados entre sí, que pretenden, fundamentalmente, responder distintas cuestiones sobre la situación descrita. En la primera actividad: describir los hechos clave referidos a la situación descrita, determinar los elementos significativos de la situación expuesta e identificar los momentos y tiempos decisivos de la situación. En la segunda actividad: identificar cuáles son las causas del problema, indicar las soluciones al problema que existe en la situación planteada, decantarse de manera justificada por la mejor solución, así como analizar las implicaciones que ello supone.

Finalmente, se incluye el producto final, que requiere, por un lado, un informe con las conclusiones del estudio de caso analizado $\mathrm{y}$, por otro, que el alumnado exponga ante el resto de compañeros las principales conclusiones que extraen de la situación analizada, mediante la defensa, el debate, o incluso la modificación de sus ideas originales, a través de la interacción que se produce con el resto de sus compañeros y de los docentes que intervienen durante la exposición.

Por tanto, durante las primeras clases presenciales, se dedicaron, aproximadamente, 5 h lectivas a la presentación y reparto de los estudios de caso por parte de los docentes a los distintos grupos de trabajo (formados por un máximo de 5 miembros), dado que era necesario que dichos grupos tuviesen claros los objetivos de aprendizaje que se deseaban lograr con el análisis de cada caso.

Una vez presentados los estudios de caso al alumnado y establecida la fecha de entrega de las diferentes actividades, durante el horario de impartición de las clases prácticas de cada una de las 3 asignaturas, se trabajó con los distintos grupos, desarrollando secuencialmente el análisis del caso de forma conjunta entre docentes y alumnado.

Finalmente, se procedía, ya de forma independiente en cada grupo, fuera del horario docente, a conceptualizar el estudio de caso, con el objetivo de encontrar una posible solución para la situación descrita, tras el análisis que anteriormente se había efectuado junto con los docentes, lo que dio lugar a la entrega de las 2 actividades periódicas.

Una vez entregadas las 2 actividades, el alumnado contaba con la capacidad de establecer las conclusiones del estudio de caso que había analizado. Es decir, los estudiantes han debido ser capaces de sintetizar lo que han descubierto durante el análisis del estudio de caso, tras haber promovido la reflexión grupal sobre los aprendizajes logrados por cada integrante del grupo. De esta forma, a través de un consenso global del grupo, deben plasmar en el informe final sus conclusiones, que estarán basadas en el análisis de la información facilitada, lo que permite establecer opiniones y juicios sobre la situación descrita.

\section{Instrumentos de evaluación}

Para evaluar en qué medida el alumnado ha adquirido estas competencias clave o transversales - tanto en la promoción piloto que ha utilizado como metodología docente el estudio de caso como en la promoción predecesora que ha utilizado sistemas tradicionales de docencia - se han considerado 2 sistemas de evaluación (Bol, Saiz y Pérez, 2013).

En primer lugar, los profesores responsables de impartir las asignaturas implicadas procedían a evaluar, de acuerdo con una plantilla diseñada al efecto (Apéndice B), la adquisición de las competencias clave o transversales en la correspondiente asignatura.

En segundo lugar, se utilizaba una valoración del tutor externo en la empresa o institución en la que el alumnado ha realizado las prácticas curriculares del plan de estudios, a través de una encuesta (Apéndice C). Con esta segunda valoración del tutor externo se pretenden 2 objetivos concretos. Por un lado, aportar una visión adicional a la visión sesgada que podría aportar el profesor, al ser este parte integrante de la metodología docente aplicada. Por otro lado, dado que las competencias clave o transversales están estrechamente relacionadas con las nuevas habilidades que se requieren a los profesionales del sector -y que mejoran su inserción en el mercado laboral-, la visión del tutor externo resulta clave en este proceso. 
Tabla 2

Estadísticos descriptivos por competencias y test F de ANOVA

\begin{tabular}{|c|c|c|c|c|c|}
\hline Ítems & $\begin{array}{l}\text { Metodología tradicional } \\
\text { M (DT) }\end{array}$ & $\begin{array}{l}\text { Metodología de estudio de caso } \\
\text { M (DT) }\end{array}$ & $\mathrm{F}$ & $\mathrm{p}^{*}$ & $\eta^{2}$ \\
\hline $\begin{array}{l}\text { Valoración global de las competencias claves o transversales } \\
\text { Competencia clave o transversal }\end{array}$ & $7,53(0,21)$ & $8,25(0,26)$ & 214,54 & $<0,001$ & 0,36 \\
\hline Adaptación a nuevas situaciones & $7,50(0,23)$ & $9,19(0,24)$ & 114,11 & $<0,001$ & 0,11 \\
\hline Aprendizaje autónomo & $7,56(0,24)$ & $8,26(0,33)$ & 96,44 & $<0,001$ & 0,13 \\
\hline Capacidad de análisis y síntesis & $7,54(0,29)$ & $8,20(0,26)$ & 87,20 & $<0,001$ & 0,12 \\
\hline Capacidad de gestión de la información & $8,01(0,33)$ & $8,13(0,26)$ & 3,98 & 0,097 & 0,01 \\
\hline Capacidad de organización y planificación & $7,58(0,21)$ & $7,51(0,22)$ & 1,09 & 0,298 & 0,00 \\
\hline Comunicación oral y escrita & $7,56(0,23)$ & $8,24(0,29)$ & 95,87 & $<0,001$ & 0,08 \\
\hline Creatividad & $7,24(0,26)$ & $8,20(0,21)$ & 101,70 & $<0,001$ & 0,10 \\
\hline Habilidades de las relaciones interpersonales & $8,22(0,27)$ & $8,26(0,29)$ & 0,24 & 0,626 & 0,00 \\
\hline Iniciativa y espíritu emprendedor & $7,60(0,26)$ & $8,23(0,26)$ & 83,52 & $<0,001$ & 0,09 \\
\hline Liderazgo & $7,59(0,20)$ & $8,13(0,20)$ & 74,43 & $<0,001$ & 0,11 \\
\hline Razonamiento crítico & $8,25(0,27)$ & $8,90(0,23)$ & 91,43 & $<0,001$ & 0,07 \\
\hline Resolución de problemas & $7,49(0,26)$ & $9,11(0,23)$ & 182,14 & $<0,001$ & 0,23 \\
\hline Toma de decisiones & $8,54(0,24)$ & $9,12(0,28)$ & 71,69 & $<0,001$ & 0,05 \\
\hline Trabajo en equipo & $7,55(0,29)$ & $9,23(0,27)$ & 257,02 & $<0,001$ & 0,42 \\
\hline
\end{tabular}

*n.s.: 0,01 .

Tanto en la evaluación efectuada por el profesor como en la encuesta realizada al tutor externo, se ha utilizado una escala con 10 niveles posibles de respuesta. Un valor de 10 significa que el alumno, de acuerdo con sus actitudes y habilidades, ha adquirido de forma muy satisfactoria la competencia evaluada, mientras que un valor de 1 significa que el alumno, de acuerdo con su conducta y los conocimientos mostrados, no muestra ninguna capacidad en la competencia evaluada.

\section{Procedimiento}

Son 3 las asignaturas implicadas en el proyecto: Estructura de Mercados (asignatura de 6 créditos impartida en el segundo curso académico), Política Económica del Turismo (asignatura de 6 créditos impartida en el tercer curso académico) y Análisis de Coyuntura Turística (asignatura de 6 créditos impartida en el cuarto curso académico). Los docentes encargados de impartir dichas asignaturas han procedido a implementar, como prueba piloto, el estudio de caso como metodología docente.

Una vez implantada esta metodología, se ha tratado de evaluar la adquisición de las denominadas competencias clave o transversales, dado que, de acuerdo con la literatura, la resolución de un estudio de caso permite desarrollar habilidades como el análisis, la síntesis y la evaluación de la información. Por tanto, posibilita también el desarrollo del pensamiento crítico, el trabajo en equipo o la toma de decisiones, además de otras actitudes y valores, como la innovación y la creatividad (Grandon, 2011; Martínez, 2006; Yacuzzi, 2005).

La Universidad de Jaén establece una serie de competencias clave o transversales que deben adquirir los futuros graduados que cursan esta titulación: adaptación a nuevas situaciones, habilidades de las relaciones interpersonales, aprendizaje autónomo, iniciativa y espíritu emprendedor, capacidad de análisis y síntesis, liderazgo, capacidad de gestión de la información, razonamiento crítico, capacidad de organización y planificación, resolución de problemas, comunicación oral y escrita, toma de decisiones, creatividad y trabajo en equipo.

Para evaluar estas competencias, al finalizar las clases (normalmente, durante la última semana docente del correspondiente cuatrimestre, para que no exista retardo entre el desarrollo de la asignatura y la evaluación efectuada por el profesor que provoque efecto olvido), los docentes encargados de impartir las asignaturas han utilizado la plantilla de evaluación a la que se ha hecho referencia en el apartado anterior. Por su parte, la encuesta realizada por el tutor externo ha sido realizada y entregada a los responsables del proyecto, una vez finalizadas las prácticas del alumno, junto con el acta de calificación de dichas prácticas, para evitar, igual que en el caso de los docentes, el efecto olvido de las capacidades del alumno.

Al tener una muestra formada por 56 participantes (32 en el grupo de control y 24 en el grupo experimental), y haber realizado 4 evaluaciones diferentes - una evaluación por cada una de las 3 asignaturas impartidas, más la encuesta realizada al tutor externo de prácticas-, el número total de observaciones para cada grupo asciende a 128 y 96, respectivamente. Se ha comprobado, antes de realizar el análisis estadístico, que no existen diferencias significativas entre las valoraciones realizadas por los profesores y las valoraciones realizadas por los tutores externos, por lo que se han analizado de forma conjunta todas las valoraciones realizadas.

\section{Análisis de datos}

El análisis de la información recabada en la presente investigación ha sido realizado a través de técnicas estadísticas inferenciales. De acuerdo con el objetivo principal de la presente investigación, se ha realizado una prueba paramétrica a través de un análisis ANOVA para comprobar si la valoración global de las competencias clave o transversales de los integrantes del grupo experimental es superior a la valoración global de dichas competencias en el grupo de control.

Además, con el objetivo de conocer qué competencias son adquiridas en mayor medida a través del estudio de caso respecto a la metodología de docencia tradicional, también se ha aplicado un test F de ANOVA de un factor de efectos fijos (Bol et al., 2013; Martín y Rodríguez, 2003) a cada una de las variables analizadas.

Se ha comprobado que el modelo inferencial que se pretende utilizar sigue una distribución normal, a través de la prueba de Kolmogorov-Smirnov, y que las varianzas del grupo de control y del grupo experimental no presentan diferencias significativas, mediante una prueba $\mathrm{F}$ para varianzas de 2 muestras. Todos los tratamientos estadísticos se han realizado con el programa SPSS 19.0 .

\section{Resultados}

Para contrastar la hipótesis planteada al inicio de este trabajo se ha utilizado un test F de ANOVA, para comprobar si existen diferencias estadísticamente significativas entre la valoración global de las competencias clave o transversales entre los 2 grupos de la muestra. Esto permite comprobar si el alumnado que ha utilizado el estudio de caso como metodología docente ha adquirido en mayor medida dichas competencias (tabla 2). 
Comprobada la diferencia de resultados entre ambos métodos docentes, con el objetivo de conocer qué competencias concretas se han adquirido en mayor medida con la metodología del estudio de caso, se ha realizado, asimismo un test F de ANOVA para cada una de las variables valoradas por los docentes y tutores externos (tabla 2).

Como puede observarse, a través de la metodología del estudio de caso se han adquirido en mayor medida la mayoría de las competencias clave o transversales definidas en el Grado de Turismo de la Universidad de Jaén. Con excepción de la capacidad de gestión de la información y de la organización y planificación de tareas, así como de las habilidades de las relaciones interpersonales, el resto de competencias clave o transversales han sido adquiridas en mayor medida mediante la metodología docente basada en el estudio de caso.

\section{Discusión}

Como se indicaba al principio del presente trabajo, el objetivo consistía en analizar si la puesta en marcha de una metodología de aula basada en el estudio de caso favorece la adquisición de las competencias transversales de la titulación en mayor medida que otras metodologías, en una muestra de alumnado del Grado de Turismo.

De acuerdo con los resultados obtenidos, se evidencia que la metodología docente basada en el estudio de caso podría mejorar el rendimiento del alumnado con relación a la adquisición de las competencias clave o transversales, por lo que los resultados apoyan la hipótesis establecida al inicio de la presente investigación.

Por tanto, la utilización del estudio de caso como metodología docente podría suponer la adquisición, en mayor medida, de las denominadas competencias clave o transversales, tal como ya habían apuntado con anterioridad otros trabajos (FUS, 2004; Universia, 2007). Esta metodología puede suponer una mejora en el proceso de aprendizaje del alumnado que ha cursado el Grado de Turismo, por lo que la mayor implicación práctica que tiene el aprendizaje basado en el estudio de caso es una mayor formación del alumnado que recibe esta metodología. Estos aumentan sus ventajas competitivas de cara a su inserción en el mercado de trabajo, dado que el sector turístico ha comenzado a demandar, como indica el Servicio Público de Empleo Estatal (SEPE, 2011), una serie de perfiles profesionales que requieren de estas competencias transversales.

Sin embargo, la metodología del estudio de caso no ha ejercido ningún tipo de influencia en la adquisición de algunas competencias concretas, de forma análoga a lo que ha ocurrido en otros trabajos previos (Díaz y Del Toro, 2013; Fernández, Maiques y Ábalos, 2012).
En concreto, la capacidad de gestión de la información, la organización y planificación de tareas y las habilidades de las relaciones personales, probablemente como consecuencia de la aplicación de un nuevo método docente aún no asimilado por el alumno, dado que este se encuentra acostumbrado a tener toda la información necesaria en un manual de referencia, en el que puede consultar las preguntas de evaluación de forma tradicional (García, 2010).

No obstante, de forma global, comparando los resultados con los obtenidos con anterioridad al desarrollo de esta experiencia (con la metodología de docencia tradicional), se ha comprobado mediante diferentes indicadores, en la misma línea que investigaciones previas sobre la evaluación de la metodología del estudio de caso (Díaz, Piñeiro, Jiménez, Palacios y Benayas, 2013; Sánchez, Santos, Fuentes y Núñez, 2015), que este método innovador para la docencia en el Grado de Turismo podría suponer mejores resultados académicos y una mayor valoración en las denominadas competencias transversales. Por tanto, pueden resultar clave en el proceso de inserción en el mercado laboral.

Sin embargo, la presente investigación cuenta con una serie de limitaciones que hay que tener en cuenta a la hora de interpretar los resultados obtenidos y que convierten el presente trabajo en un estudio exploratorio. En primer lugar, al tratarse de un diseño cuasiexperimental, no se tiene control sobre todas las variables relevantes que podrían influir en la mejora de los resultados obtenidos por parte del alumnado (por ejemplo, innovaciones impulsadas desde otras asignaturas, una mayor capacidad intelectual o mayores habilidades de los integrantes del grupo experimental), por lo que la diferencia entre los 2 grupos también podría deberse a estas variables. En segundo lugar, los profesores que han diseñado e implementado el estudio de caso como metodología docente son los mismos docentes que han evaluado las competencias, por lo que podría existir algún tipo de sesgo en la evaluación realizada. Finalmente, se ha utilizado una muestra concreta, formada por el alumnado del Grado de Turismo de la Universidad de Jaén, por lo que la generalización de los resultados a otros contextos debe realizarse con precaución.

La experiencia descrita indica que la metodología del estudio de caso aplicada al Grado de Turismo de la Universidad de Jaén mejora la adquisición de competencias transversales por parte del alumnado que cursa dicho grado, respecto a los métodos de docencia tradicionales.

\section{Conflicto de intereses}

Los autores declaran no tener ningún conflicto de intereses. 
Apéndice A. Ejemplo de actividad empleada en el aula

Departamento de Economía

Universidad de Jaén
Grado en Turismo

Política Económica del Turismo

ASIGNATURA: Política Económica del Turismo. Grado de Turismo. $3^{\circ}$ curso

PREGUNTA MOTRIZ: ¿Podrá seguir manteniendo España su actual posicionamiento competitivo como destino turístico mundial en las próximas décadas?

ESCENARIO: Acabada su formación universitaria y su período de prácticas, le acaba de contratar una de las consultoras turística de mayor prestigio en España. En su primera reunión de trabajo, con el resto del equipo, el Jefe de Proyectos y Programas presenta el proyecto en el que Vd. va a trabajar. Un encargo del Gobierno para el diseño de una agenda estratégica que contribuya a consolidar España como destino turístico mundial.

La Organización Mundial del Turismo ha facilitado sus previsiones de evolución de la demanda turística a nivel mundial hasta el año 2030. Actualmente, España es el segundo destino turístico mundial por volumen de ingresos, pero en los próximos años se va a producir una redistribución de los flujos turísticos entre Europa y Asia Pacífico, que no beneficia, precisamente, a España.

$$
\text { ooo0ooo }
$$

ACTIVIDAD 1: Análisis de la evolución de la demanda turística internacional desde 1950 y discusión de las previsiones de la OMT para 2030. Hay que prestar especial atención a dos cuestiones: i) el papel que juega España en todo este proceso; ii) el cambio producido en el panorama mundial de destinos-país durante estos sesenta años.

ENTREGABLE 1: Documento EXCEL en el que se recoja el análisis realizado, así como un documentos en (Word o PDF) con las previsiones realizadas por ellos y sus principales conclusiones respecto al análisis propuesto.

$$
\text { ooo0ooo }
$$

ACTIVIDAD 2: Como miembro de esta consultora que Vd. es, ¿qué acciones propondría con el fin de mantener a España en el Top5 de destinos turísticos del mundo en 2030?

ENTREGABLE 2: Documento que incluya, al menos, cinco líneas estratégicas, justificadas, con establecimiento de objetivos y desarrolladas con sus correspondientes acciones, actores implicados, nivel de prioridad, etc.

$$
\text { ooo0ooo }
$$

PRODUCTO FINAL: Cada grupo deberá entregar por escrito y presentar en clase (presentación oral con apoyo multimedia elegido por cada grupo) un informe final que contendrá sus conclusiones acerca de la pregunta planteada, así como las actuaciones que, a su juicio, España deberá desarrollar en los próximos años como destino turístico para posicionarse en la línea que cada grupo defienda. (La presentación debe durar 10 minutos aproximadamente. Este informe será discutido y evaluado en una sesión de debate realizada entre todos los grupos). 


\section{Apéndice B. Plantilla para evaluar la adquisición de competencias por parte del profesor}

Asignatura:

Nombre del alumno:

Profesor responsable:

Fecha de la evaluación:

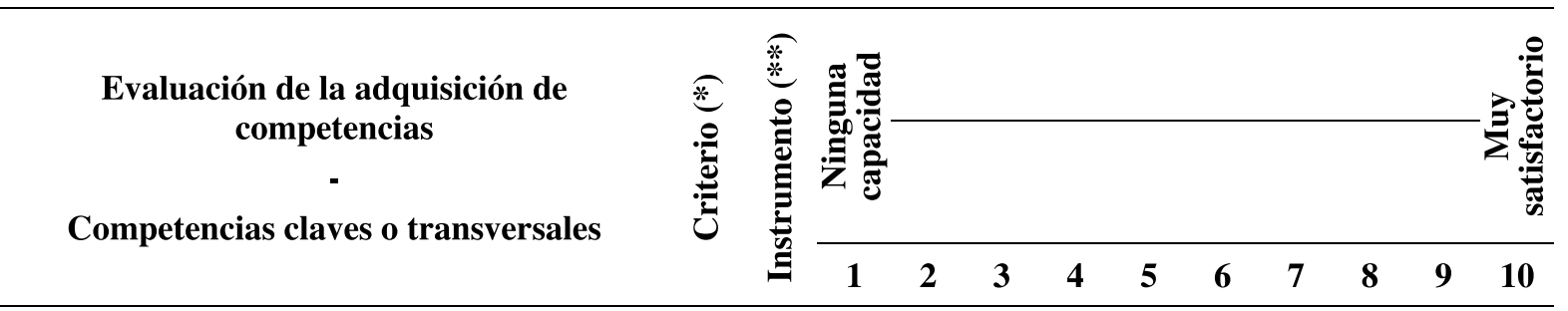

Adaptación a nuevas situaciones

Aprendizaje autónomo

Capacidad de análisis y síntesis

Capacidad de gestión de la información

Capacidad de organización y planificación

Comunicación oral y escrita

Creatividad

Habilidades de las relaciones interpersonales

Iniciativa y espíritu emprendedor

Liderazgo

Razonamiento crítico

Resolución de problemas

Toma de decisiones

Trabajo en equipo

\section{Valoración global adquisición competencias}

(*) Criterio utilizado para evaluar la competencia:

A - Dominio de los conocimientos teóricos, prácticos y operativos de la asignatura

B - Vinculación de las conclusiones aportadas con los datos obtenidos en la fase de análisis

C - Defensa y exposición del correspondiente informe entregado

(**) Instrumento de valoración utilizado para evaluar la competencia:

A - Análisis y revisión de las actividades entregadas

B - Estudio del informe entregado, valorando especialmente los argumentos utilizados

C - Observancia del profesor en la exposición realizada así como en las clases prácticas 


\section{Apéndice C. Encuesta para evaluar la adquisición de competencias por parte del tutor externo de prácticas curriculares}

Nombre del alumno:

Empresa o institución:

Tutor responsable:

Fecha de la encuesta:

\section{Evaluación de la adquisición de competencias}

\section{Competencias claves o transversales}

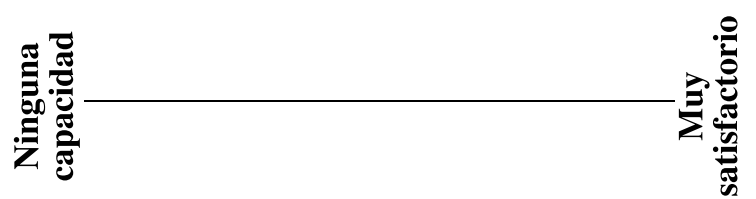

\section{$\begin{array}{llllllllll}1 & 2 & 3 & 4 & 5 & 6 & 7 & 8 & 9 & 10\end{array}$}

Adaptación a nuevas situaciones

Aprendizaje autónomo

Capacidad de análisis y síntesis

Capacidad de gestión de la información

Capacidad de organización y planificación

Comunicación oral y escrita

Creatividad

Habilidades de las relaciones interpersonales

Iniciativa y espíritu emprendedor

Liderazgo

Razonamiento crítico

Resolución de problemas

Toma de decisiones

Trabajo en equipo

\section{Valoración global adquisición competencias}

\section{Referencias bibliográficas}

ANECA - Agencia Nacional de Evaluación de la Calidad y Acreditación (2004). Título de Grado en Turismo. Libro Blanco. Madrid: ANECA.

Bol, A., Saiz, M. C. y Pérez, M. (2013). Validación de una encuesta sobre la actividad docente en educación superior. Aula Abierta, 41(2), 45-54.

CGC - Club de Gestión de la Calidad (1998). Mejora en la formación universitaria: Sugerencias desde la empresa. Madrid: CGC.

Díaz, M. J., Piñeiro, C., Jiménez, A., Palacios, M. y Benayas, J. (2013). El aprendizaje social percibido en los procesos de participación. Estudio de caso de las estrategias de educación ambiental en España. $M+A$. Revista Electrónica de Medioambiente, 14(2), 1-16.

Díaz, R. T. y del Toro, P. S. (2013). Estrategias de intervención para la formación de competencias profesionales en la educación superior. Pedagogía Universitaria, 15(5), 39-53.

FCD - Fundación Conocimiento y Desarrollo (2004). La contribución de las universidades españolas al desarrollo. Madrid: FCD.

Fernández, A., Maiques, J. M. y Ábalos, A. (2012). Las buenas prácticas docentes de los profesores universitarios: estudio de casos. Revista de Docencia Universitaria, 10(1), 43-66.

FUS - Fundación Universidad-Sociedad (2004). Programa de adaptación a la empresa. Programa integral de preparación para la adaptación e integración en la empresa de titulados con convenios en prácticas. Sevilla: FUS.

García, J. (2010). De profesor tradicional a profesor innovador. Temas para la Educación, 11, 1-10.
Grandon, T. (2011). Book Informing with the case method. London: Informing Science Press.

Martín, A. V. y Rodríguez, M. J. (2003). Estilos de aprendizaje y grupos de edad: comparación de 2 muestras de estudiantes jóvenes y mayores. Aula Abierta, 32, 97-114.

Martínez, P. C. (2006). El método de estudio de caso. Estrategia metodológica de la investigación científica. Pensamiento y Gestión, 20, 165-193.

Moen, T.(2006). Reflections on the narrative research approach. International Journa of Qualitative Methodology, 5(4), 56-69.

Oliveira, M., Mazera, S. M., Guillaumon, M. L. y Fernández, E. (2014). Análisis de la producción científica en Brasil sobre dificultades de aprendizaje: una revisión bibliométrica. Aula Abierta, 42(1), 31-38.

Sánchez, S. M., Santos, L., Fuentes, F. J. y Núñez, J. M. (2015). Enseñanza-aprendizaje por competencias en la educación superior. La construcción de casos de empresa. Educación XXI, 18(1), 237-258.

SEPE - Servicio Público de Empleo Estatal (2011). Observatorio de las ocupaciones. Características sociodemográficas y perfiles competenciales de los trabajadores del sector turismo. Madrid: SEPE.

Universia (2007). Las competencias profesionales en los titulados. Contraste y diálogo Universidad-Empresa. Madrid: Universia.

Yacuzzi, E. (2005). El estudio de caso como metodología de investigación: teoría, mecanismos causales, validación. Inomics, 1, 296-306. 